\title{
Thermography data fusion for failure analysis
}

\author{
by A. Gleiter, C. Spiessberger, and G. Busse
}

\author{
Institute of Polymer-Technology - Non-Destructive Testing - University of Stuttgart
}

\begin{abstract}
Non-destructive evaluation is important both for manufacturing and maintenance inspection of safetyrelevant structures most of which relate to aerospace applications. Besides detection of subsurface boundaries in such structures it is essential to characterise them e.g. with thermography methods which provide the unique chance that different aspects of defects can be imaged depending on the choice of the excitation source. As such images are taken with the same camera from the same angle of view, images can be compared or merged with no need for corrections of topography. With this way of feature extraction the nature of defects can be described more accurately.
\end{abstract}

\section{Introduction}

With optical excitation of thermal waves, heat is deposited mostly at the surface of the inspected structure from where thermal waves propagate to be reflected from internal boundaries back to the surface where they interfere with the incident wave. Images extracted from thermographic data or stacks of images display boundaries almost regardless of how well both sides of such boundaries stick to each other.

With thermography monitoring the sample during ultrasound injection, areas with higher losses have a strong response since they generate more heat during each cycle of loading in the alternating ultrasound stress field. So the corresponding image displays - at least partially - the imaginary part of Young's modulus which describes a mechanical property. Therefore such images reveal features that are otherwise seen only when ultrasound damping is used for imaging.

So different aspects of the same sample and its defects are recorded with the same thermographic camera. Therefore, such images are well suited to perform data fusion and feature extraction so that the nature of the defect can be more clearly identified. This is important information for people who try to predict the effect of defects under a given load until final failure. This paper does not go into the complicated problem of predicting life time under a given load scenario for a given defect. It only demonstrates data merging and the additional information derived from such images.

\section{Measurement Setup}

The measurement setup was the one being used for standard Lockin-Thermography based on optical or ultrasound excitations (figure 1). In this case, the sample could be excited either by halogen lamps or by an ultrasound transducer which was the sample mount. This way the pixels of the images corresponded exactly to the same locations on the sample. This arrangement is an advantage compared to combination of other methods where different angles of imaging have to be used so that sample topography needs to be measured as well in order to match the pixels.
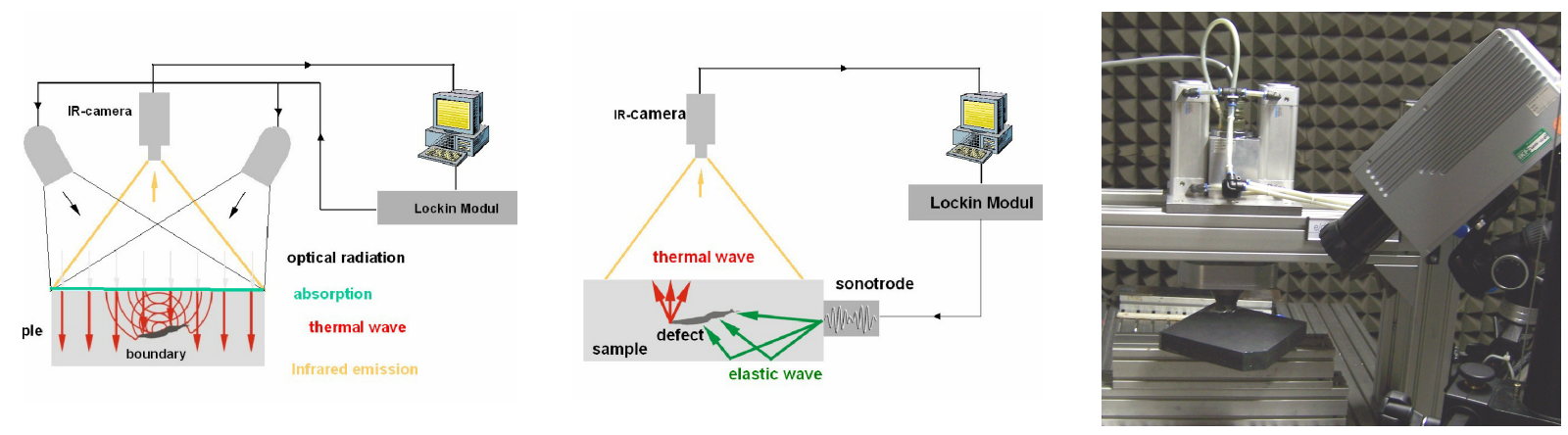

Fig. 1: Lockin-Thermography with optical (left) or ultrasound excitation (middle: principle, right: set up)

\section{Results}

The following examples have been selected since their inspection is important for aerospace materials. One material is $\mathrm{C} / \mathrm{CSiC}$ which is fabricated from carbon fibre laminate by heating and subsequent infiltration with liquid silicon at $1500 \mathrm{deg}$ Celsius so each carbon fibre is surrounded by a SiC tube with very high strength. Therefore this material is being used e.g. for re-entry structures of spacecraft and for high speed brakes of vehicles and aircraft. The defects to be detected can be inhomogeneous infiltration or disbond of layers. 


\section{Results on C/C-SiC}

The result displayed in figure 2 has been obtained on a C/CSiC plate containing an area with a different silicon content resulting from wrong conditions during manufacturing. This area is revealed by optically excited Lockin-Thermography (OLT, left) responding to local variations of thermal diffusivity. Ultrasound LockinThermography (ULT, right) performed on the same sample responds to local conversion of elastic energy into heat which occurs mostly in cracks due to friction effects. Therefore the ULT-image shows selectively cracks while OLT responds to any boundary. The advantages of both methods are combined by data fusion performed here simply by superposition of both images (middle): It becomes evident that the boundary between the two areas of different silicon content is the source of crack formation. The reason is in this case different thermal expansion which caused crack initiation at the boundary due to shear load when the sample cooled down after the high-temperature production process. So data fusion provides the full information about the defect and how it results in failure.
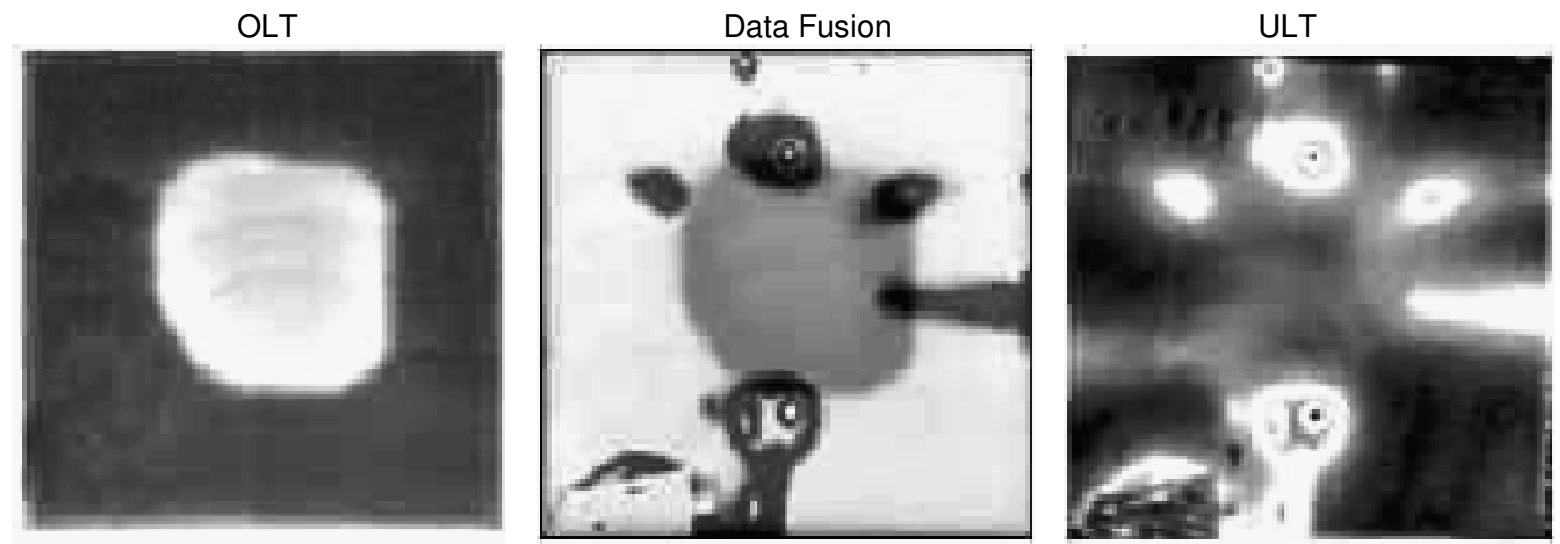

Fig. 2: Silicon-carbide (C/CSiC) block containing an area of different Si infiltration. OLT displays the thermal boundaries and ULT the mechanical losses due to friction heating. Data merging (middle) shows that crack formation starts at the boundaries revealed with OLT.

Another example for the same kind of material is displayed in figure 3. Here the defect is a delamination extending from one edge. The thermal discontinuity is revealed by OLT, a slight edge-effect responding to lateral heat flow is visible as well. ULT shows that the boundary has some structure. To avoid standing wave effects as artefacts the ultrasound frequency was modulated. So the bright spots indicate point contacts that heat up by rubbing in the ultrasound field. So the combined information derived from the two images (middle) is that the thermal boundary is not flat but that it has some contact bridges across it. The distortion of the delamination front just at two contacts raises the question which role these two spots played in the generation of the delamination.
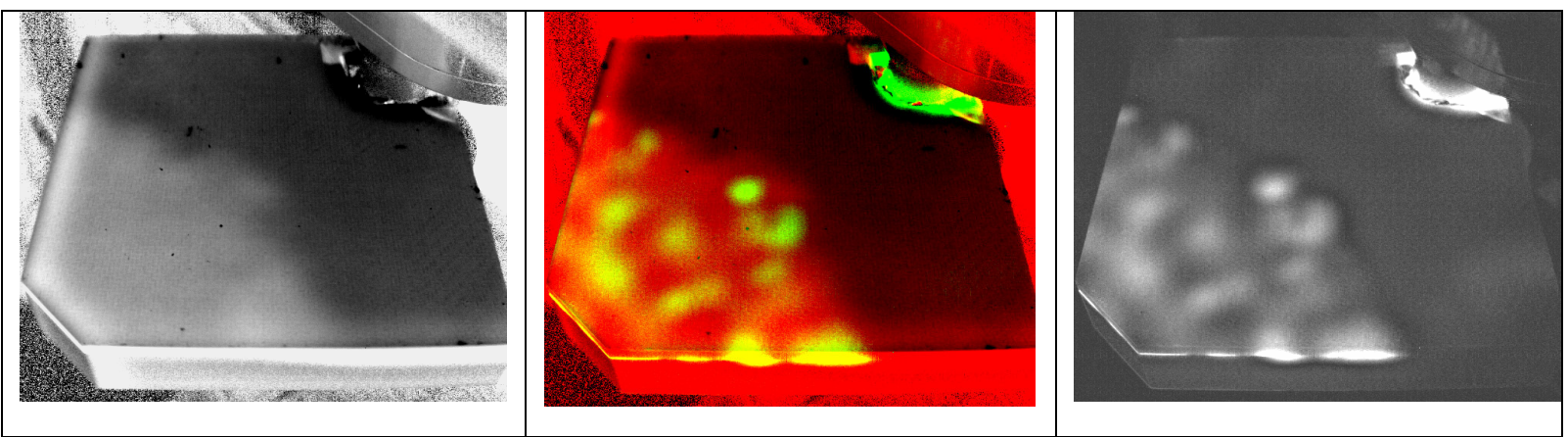

Fig. 3: OLT phase image (left, Lockin-frequency $0.1 \mathrm{~Hz}$ ) and ULT amplitude image (right, Lockin-frequency $0.2 \mathrm{~Hz}$, frequency modulation $15-25 \mathrm{kHz}$ ). Bright spot at top right edge is the active ultrasound transducer. Data merging (middle) shows how the point contacts are located within the delamination. 


\section{CFRP damage due to jet cutting process}

Carbon fibre reinforced polymers (CFRP) have a very high specific strength which makes them attractive to applications where low weight is required. In many cases the final shape of the component is cut from a laminate. In such cases the jet cutting process itself with the high mechanical load can cause disbonds whose size is unpredictable. The start of the jet cutting process is obviously the most critical moment as is revealed by the OLT results in figure 4. Though the geometry of all cut-outs is the same, disbonds of variable sizes form around the starting points. These bright points are the dominant features even after the cutting process. Surprisingly they display much stronger friction than the edges of the delaminations where the transition to intact material occurs. The edges along the cut-outs display minor boundary friction within a certain distance. Such results should help to clarify the mechanisms of damage during cutting and to develop parameter constellations where such damage is reduced.

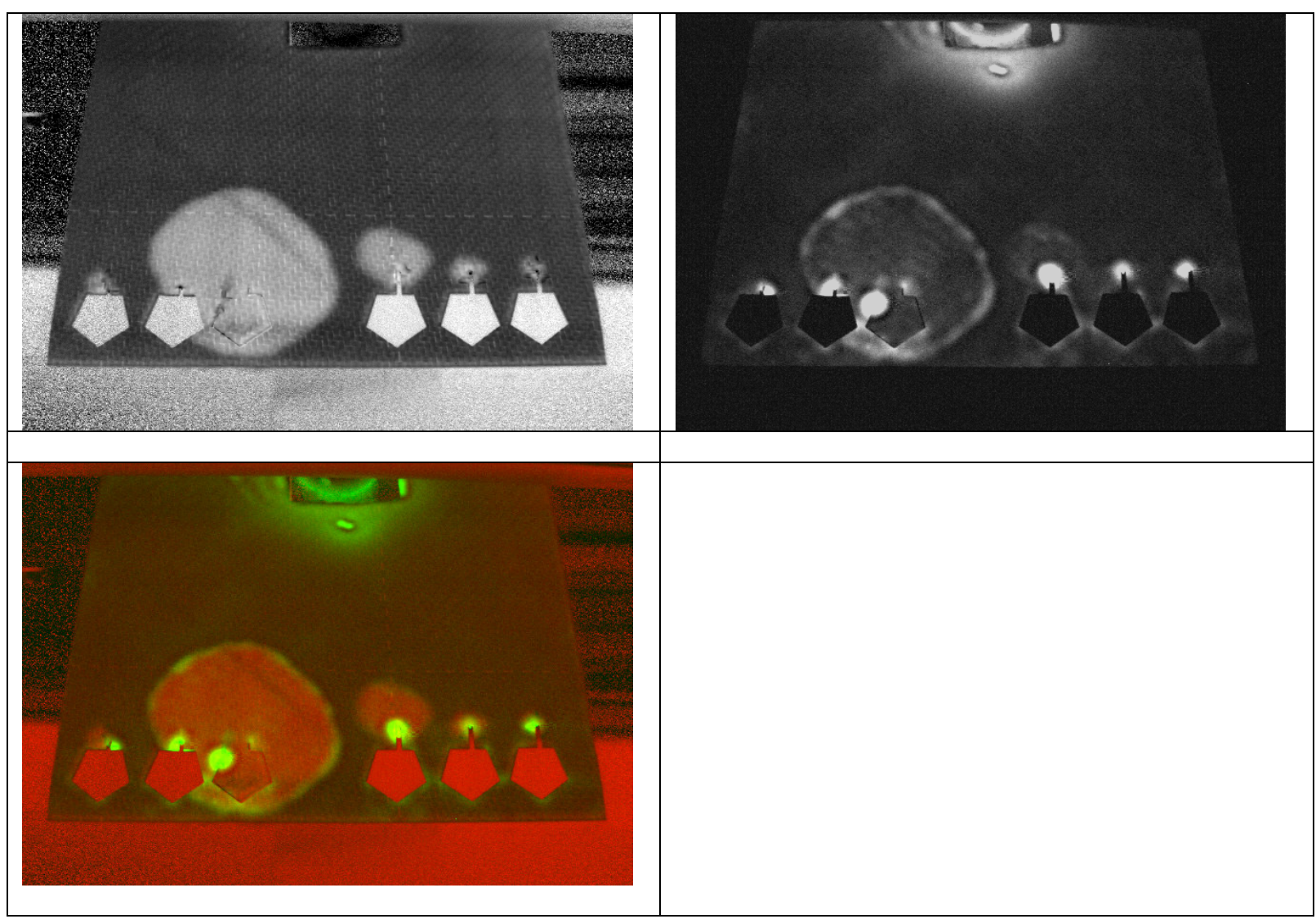

Fig. 4: Carbon fibre reinforced laminate plate with cut-outs. OLT phase image (top left, Lockin-frequency $0.5 \mathrm{~Hz}$, and ULT-FM amplitude image (top right, Lockin-frequency $0.2 \mathrm{~Hz}$ ) together with data fusion image (bottom left).

\section{Conclusions}

The examples presented above indicate that the understanding of damage development can be improved by data merging. This is important for the safe use of modern materials. As a next and obvious extension of such comparisons it should be of interest to perform data fusion on a pixel-base, like it has been done some time ago for other measurement data [1]. This way a new kind of images [2] could be generated that display directly the kind of defect without the need of a human observer. So this technique is relevant for the development of reliable automated systems to identify critical defects during production or maintenance inspection.

\section{REFERENCES}

[1] G. Busse, B. Bruhl, L. Diender, P. Elsner, and M. Ota. Neue Methoden der zerstörungsfreien Prüfung für Polymerwerkstoffe. DVM, Berlin (1990) 261-276

[2] C. Spiessberger, A. Gleiter, and G. Busse. Data fusion of Lockin-Thermography Phase Images (this QIRT conference) 
http://dx.doi.org/10.21611/qirt.2008.12_02_16 